\title{
What is the evidence for the change in the blood donation deferral period for high-risk groups and does it go far enough?
}

\author{
Authors: Beattie RH Sturrock ${ }^{A}$ and Stuart Mucklow ${ }^{B}$
}

In November 2017, the deferral on blood donations from highrisk groups in Great Britain was changed to 3 months from last at-risk sexual contact following recommendations from the Advisory Committee on the Safety of Blood, Tissues and Organs. This represented a reduction from 12 months for men who have sex with men, and from a lifetime ban for sex workers. This is a step forward for equality and for reducing stigma around these groups. However, one argument for deferral is the prevalence of infections, which may not be identified due to the fallibility of current testing approaches. Clearly it is vital that the welfare of blood transfusion recipients is prioritised and they are not exposed to unacceptable risks. However, with the increasingly sophisticated technology used to screen blood, it can be argued that the evidence shows that the reduction in deferral does not go far enough.

KEYWORDS: MSM, blood donation, NAT, HIV, deferral

\section{Introduction}

The UK blood transfusion services rely on voluntary blood donation and exclusion of potential donors can be a controversial and emotive topic. Prior to being allowed to donate blood, a potential donor must undergo a screening questionnaire, ${ }^{1}$ which includes questions pertaining to their sexual practices. Responses indicating potentially unsafe blood may result in deferral. Permanent deferral of high-risk groups, including men who have sex with men (MSM), was initially introduced in the 1980s with the emergence of acquired immunodeficiency syndrome (AIDS). At this time, these precautions were deemed necessary due to the lack of testing for the AIDS pathogen, until HIV was identified in 1984. ${ }^{2}$ In 2011, the regulations were amended to allow MSM to donate if 12 months had elapsed since their last at-risk sexual contact. ${ }^{3}$ In July 2017, the government announced its intention to reduce the deferral of MSM as well as commercial

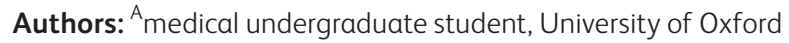
Medical School, Oxford, UK; ${ }^{\mathrm{B}}$ Clinical haematology consultant, Department of Clinical Haematology, Royal Berkshire Hospital, Reading, UK sex workers (CSW) and those with a partner who has a high risk of an infection which could be sexually transmitted, or who has been sexually active in a high HIV prevalence region. ${ }^{4}$ This deferral period came into practice in Great Britain in November 2017 and reduced the time between last sexual contact and donating blood to 3 months. ${ }^{5,6,7}$ This is a change from a 12 -month deferral for people who have a high-risk partner, and from a lifetime ban for $\mathrm{CSW}^{5}$ The decision to reduce the deferral period was based on recommendations from the Advisory Committee on the Safety of Blood, Tissues and Organs (SaBTO). These were based on a variety of factors including that routine surveillance found that the reduction from permanent to 12-month deferral in 2011 did not increase the risk of transmission of blood-borne infections. ${ }^{8}$

The benefits of the reduction are numerous, not least equality for these groups, although there is still an obvious disparity in the fact that heterosexual individuals and women who have sex with women, are not deferred (unless they have a high risk partner or exchange sex for money). Furthermore, deferrals of MSM do not distinguish between those who may be lower risk such as those in monogamous relationships. ${ }^{9}$

However, there will always be debate about any change that could allow potentially higher risk populations to donate more freely. Although the reduction in the deferral will result in more people being eligible to donate, there is a trend to decreased use of red blood cells transfusions, ${ }^{10}$ and therefore the risks may outweigh the benefits. Currently, the risk of infection from transfusion is extremely low at 1 in 6.7 million for HIV, 1 in 1.3 million for hepatitis B (HBV) and 1 in 28 million for hepatitis $C$ (HCV). ${ }^{11}$ The safety of the patient is paramount and therefore it has been stated that the blood service should err on the side of caution. ${ }^{12}$ However, with the advances that have been made in the detection of pathogens in blood products, is there any reason to assume that the proposed change to the deferral should affect blood safety at all? Could, in fact, this change not be going far enough?

\section{How high risk are high-risk groups?}

A defence of restrictions on MSM and CSW donating blood is that they may have a greater incidence of transfusion transmissible infections (TTIs). Due to the 1980s AIDS epidemic, HIV is often associated with, although of course not confined to, MSM. In the UK, the estimated HIV prevalence in the general population in 2016 was 
1.6 per 1000; in MSM it was 58.7 per $1000 .^{13} \mathrm{CSW}$ are estimated to be 10 times more likely to contract HIV than the general population, and the spread of HIV among MSM, CSW and other high-risk groups (and their sexual partners), accounted for $36 \%$ of all new HIV infections in $2015 .^{14}$ It is worth noting that $74 \%$ of new cases therefore did not come from these groups. In addition, HIV is not the only TTI that needs to be considered, for example hepatitis A, HBV, $\mathrm{HCV}^{15}$ and syphilis ${ }^{16}$ also have a high prevalence in MSM.

In 2015, a systematic review by De Buck et al investigated the link between MSM blood donors and TTIs, and found few high quality studies to draw from. Three studies showed that there was significant correlation between MSM blood donors and HIV infection, while studies investigating HBV and HCV found no demonstrable correlation. ${ }^{17}$

In their 2017 report, SaBTO used modelling studies to convey the change in the risk of infectious donations being missed by screening in the UK in the course of 1 year with the changes in donor criteria. They estimated that the risk of potentially infectious blood donations not being detected was between 0.180.67 per million donations for HIV, and between $0.04-0.19$ per million donations for HCV. The SaBTO working group had agreed that the increase in risk that was considered acceptable was less than 1 per million. Both the increase for HIV and HCV were below this threshold, meaning they were an acceptable increase in risk. ${ }^{18}$

\section{Ensuring blood safety}

Several pathogens may be contracted through contaminated blood including HIV, HBV, syphilis, HCV, malaria $^{19}$ and variant Cretzfeld-Jakob. ${ }^{20}$ It is mandatory to test every blood donation for HIV, HBV, HCV and syphilis. In England it is mandatory to use nucleic acid amplification testing (NAT) for HCV RNA, although NAT for HIV RNA and HBV DNA is often also carried out, as the commercially available tests are triplex assays. NAT testing for HIV, HCV and HBV is performed on pools of up to 48 samples. ${ }^{21}$

The time between infection and detection by testing is called the window period (WP) and any donation made in this time would give a false negative result. Serological tests rely on seroconversion or detection of pathogen surface proteins, while NAT detects the viral genome, which is measureable soon after infection and before seroconversion. ${ }^{22}$ A 2015 study found that NAT detected HBV infected blood donations in a pool of blood samples ruled negative by seroconversion-detecting immunoassays. ${ }^{23}$ Therefore, NAT allows detection of viraemia at an earlier stage and, in doing so, reduces the WP.

For HIV, the WP with individual donation NAT for HIV is 5 days (compared to 15 for serological testing); for $\mathrm{HCV}$ is $3-5$ days (compared to 65); and for HBV it is 21 days (compared to 36). ${ }^{24}$ Understandably, this reduces the residual risk of TTIs. For example, since the introduction of NAT in France, the residual risk of HCV transmission has fallen from 0.64 per million donations to 0.1 per million donations. ${ }^{25}$

However, testing pooled samples, as is done in the UK, reduces the sensitivity of the test, ${ }^{21}$ and so the true WPs are likely to be slightly higher. Nonetheless, it is possible to detect HIV, $\mathrm{HCV}$ and HBV within 1 month of infection. It therefore follows that the 12-month deferral period is not evidence-based and perhaps 3 months is overly cautious. However, the SaBTO report notes that the international standard is a deferral period that is double the window period. ${ }^{8}$ Additionally, a cautious approach is understandable in the context of patients becoming infected through blood transfusion (such as is the subject of the Penrose inquiry). ${ }^{26}$

However, WP donation is not the only means by which infected blood could reach a patient. Infected donations can be released due to false negative errors or quarantine release errors (QRE). A study using quantitative probabilistic modelling estimated how many more MSM would become eligible if the then permanent exclusion was revised, and used this data along with disease prevalence statistics to determine how many more infected units would be donated. This, along with the rate of QREs occurring, was used to estimate the increase in erroneously released infected units. Their model suggested that a change from permanent deferral to 1-year deferral would result in an average of 0.18 and 0.02 additional HIV and HBV infected units being released per year, respectively. This represented a $3 \%$ increase in relative risk of erroneous release for HIV infected blood, and $0.04 \%$ increase for HBV infected blood. This suggests that allowing high-risk groups to donate more freely would increase infected blood being erroneously released. ${ }^{27}$ However, as the risks involved are already small, the overall increase in risk may be negligible.

\section{The importance of donor adherence}

It is possible that the reduction in deferral period could increase blood safety further as it promotes equality and is easier to adhere to, increasing compliance. Non-compliance refers to a donor not disclosing a risk behaviour that would otherwise result in them being deferred, such as recent same-sex sexual contact in the case of MSM.

A number of studies have found that a proportion of MSM were interested in donating despite not fulfilling criteria, or had already demonstrated non-compliance, which could put patients at risk. $^{28,29}$

When assessing compliance, it is important to consider why people choose to donate to understand what could precipitate an act of non-compliance. A systematic review in 2013 suggested that people choose to donate because it makes them feel good. ${ }^{30}$ This may help to explain why people choose to be noncompliant, as the donor is acting in a way that makes them feel good because it is a 'good thing to do' rather than necessarily considering the negative consequences for others. Reasons given for non-compliance are wide ranging; one study found that the reasons included the participants knowing their HIV status, practising safe sex, or feeling that the exclusion was unfair and discriminatory. ${ }^{31}$

It has been proposed that compliance with deferral is the major factor in overall blood safety, rather than the deferral period itself. Therefore, if the deferral period is shorter, but well-adhered to, then the donations may be safer than a longer deferral period that people find it harder, or are less willing, to adhere to. With the reduction of the deferral period to 3 months, not only do more people become eligible but it may also result in less noncompliance in protest. $^{32}$

\section{Alternatives to deferral}

The 3-month deferral is supported by evidence but highrisk groups, such as MSM, are still being partially excluded. A solution to this might be introducing an individual risk-behaviour 
assessment, which is blind to sexual orientation, as has been implemented in various countries including Spain and Italy. ${ }^{33}$

Italy changed from permanent deferral of MSM to an individual risk assessment in 2001. Under this system, the donor completes a questionnaire about their sexual behaviour and undergoes a confidential face-to-face interview with a doctor to discuss the donor's eligibility, regardless of their sexual orientation. The donor could be deemed no risk and so is eligible to donate, 'risk' (for example has a new sexual partner with unknown sexual behaviour) and is deferred for 4 months, or 'high risk' (for example has sex with more than one partner with unknown sexual behaviour) and is permanently deferred. A comparison of the blood donor epidemiology between 1999 and 2009/10 showed no significant change in HIV prevalence in either first time or repeat donors. However, there still appears to be a problem with non-compliance in using this system. Of the 218 blood donors who were confirmed to be HIV positive in this study who were interviewed, $35.5 \%$ reported a sexual risk exposure more than 4 months before donation, $28.5 \%$ no risk exposure and $36.0 \%$ a sexual risk exposure in the 4 months prior to donation. The reasons for the non-compliance included not believing, or underestimating, the risk of their behaviour, and thinking that the screening of their blood would detect infection. ${ }^{34}$ This conclusion was echoed by a more recent study, which investigated 349 HIV positive donors between 2009 and 2011. Of these, 32.4\% who had denied risk behaviours at the screening interview, reported risk behaviour in the 4 months prior to donating blood, with the reasons for not reporting this at the predonation screening being that they did not realise that their behaviour had been risky, thinking that the behaviour did not put them at significant risk of infection, using the donation as a way to be screened for HIV, not knowing that their partner was HIV positive, and not understanding the information they were given before donation. $^{35}$

Additionally, a study on HIV prevalence in blood donors in Spain showed that the rate among first-time blood donors was 10.3 per 100,000 donors compared to the general population incidence of 10 per 100,000 individuals. The authors suggest this correlation in rates is evidence that the methods used to screen blood donors 'have limited effectiveness'. ${ }^{36}$

A different means of deferral reduction is predonation blood screening. This would counteract the increased risk of processing errors by reducing the prevalence of infected blood in the testing pool. Potential donors have a blood sample taken for screening, and TTI-negative candidates are invited back to give a full donation. A retrospective study in the Netherlands investigated the efficacy of this system on 176,716 candidate donors (those who had never donated blood before) between 2009 and 2013. They found that $0.062 \%$ of the predonation screening samples were positive for infections, and that $82 \%$ of the candidate donors gave a donation after being invited back following a negative test. Of these subsequent donations, one was positive for HIV (and likely in the WP for infection at the time of predonation screening). Overall the use of predonation screening, prevented 110 TTI infected donors from giving blood and prevented one WP HIV donation. This system could be utilised in the UK to show which individuals are safe to provide full donations, whether they belong to a high-risk group or not. However, this system could result in the loss of donations; in the Dutch study, $18 \%$ of candidate donors did not return to complete their donation. ${ }^{37}$

\section{Conclusion}

It is clearly paramount for the UK blood transfusion services to maintain patient safety. For as long as there is a reliance on voluntary donations, and the inherent risks associated with this, there must be a balance between allowing people to donate and ensuring that blood is safe. Blood testing for infectious agents is fallible. The arrangements of the 3 -month deferral period can therefore be justified.

The gold standard may be to use a combination of individualised risk assessment and predonation screening; however, this would require a large scale change in the infrastructure of UK transfusion services, and would likely result in problems of its own, namely increased cost, time and greater complexity of operation (which could increase error).

Overall, the evidence supports the change to the 3-month deferral for groups such as MSM and CSW. This change promotes equality and may increase adherence, as well as increasing the number of eligible donors. However, this restriction is still conservative and with further high-quality evidence, the deferral period could potentially be shortened.

\section{References}

1 NHS Blood and Transplant. What happens on the day. www.blood. co.uk/the-donation-process/what-happens-on-the-day/ [Accessed 27 September 2017].

2 Wilson K, Atkinson K, Keelan J. Three decades of MSM donor deferral policies. What have we learned? Int J Infect Dis 2014;18:1-3.

3 Watkins NA, Brailsford S, Kelly D. SaBTO review of blood donor selection criteria related to sexual behaviour. Transfus Med 2011;21:363-4.

4 NHS Blood and Transplant. Scientific review offers chance for more people to give blood. 2017. www.blood.co.uk/news-andcampaigns/news-and-statements/scientific-review-offers-chancefor-more-people-to-give-blood/ [Accessed 27 September 2017].

5 Blood Donation. Blood donation rules have changed. www.blood. co.uk/news-and-campaigns/news-and-statements/blood-donationrules-have-changed/ [Accessed 8 March 2018].

6 The Scottish National Blood Transfusion Service (SNBTS). Changes to donor deferral criteria will allow more people the opportunity to give blood. www.scotblood.co.uk/news/changes-to-donor-deferralcriteria-will-allow-more-people-the-opportunity-to-give-blood/ [Accessed 10 May 2018].

7 Welsh Blood Service. More People in Wales to be eligible to donate blood. www.welsh-blood.org.uk/more-people-in-wales-to-be-eligibleto-donate-blood/ [Accessed 10 May 2018].

8 Advisory Committee on the Safety of Blood, Tissues and Organs (SaBTO). Donor Selection Criteria Report (2017). SaBTO, 2017.

9 Hurley R. Bad blood: gay men and blood donation. BMJ 2009;338:b779.

10 NHS Blood and Transplant. NHS Blood and Transplant Annual Report and Accounts 2016/17. William Lea Group, 2017.

11 Bolton-Maggs PHB, Cohen H. Serious Hazards of Transfusion (SHOT) haemovigilance and progress is improving transfusion safety. Br J Haematol 2013;163:303-14.

12 Franklin IM. Is there a right to donate blood? Patient rights; donor responsibilities. Transfus Med 2007;17:161-8.

13 Kirwan PD, Chau C, Brown AE, Gill ON, Delpech VC. HIV in the UK 2016 Report. London: Public Health England, 2016.

14 UNAIDS. Prevention Gap Report. Geneva: UNAIDS, 2016.

15 Centers for Disease Control and Prevention. Gay and Bisexual Men's Health, Viral Hepatitis. Atlanta: CDC, 2016. www.cdc.gov/ msmhealth/viral-hepatitis.htm [Accessed 27 September 2017]. 
16 Mayer K. Sexually transmitted diseases in men who have sex with men. Clin Infect Dis 2011;53:S79-83.

17 De Buck E, Dieltjens T, Compernolle V, Vandekerckhove P. Is having sex with other men a risk factor for transfusion-transmissible infections in male blood donors in western countries? A systematic review. PLOS ONE 2015;10:e0122523.

18 Katz M, Brailsford S, Reynolds C, Davison K. Modelling the risk of revised selection criteria for blood donors. Donor selection report, Advisory Committee on the Safety of Blood, Tissues and Organs (SaBTO), 2017.

19 Choudhury N. Transfusion transmitted infections: How many more? Asian J Transfus Sci 2010;4:71-2.

20 Llewelyn C, Hewitt P, Knight R et al. Possible transmission of variant Creutzfeldt-Jakob disease by blood transfusion. Lancet 2004;363:417-21.

21 Joint United Kingdom (UK) Blood Transfusion and Tissue Transplantation Services Professional Advisory Committee. Guidelines For The Blood Transfusion Services In The United Kingdom. 8th edn London: The Stationary Office, 2013:9.1 General Requirements. www.transfusionguidelines.org/red-book/chapter-9-microbiologytests-for-donors-and-donations-general-specifications-for-laboratorytest-procedures/9-1-general-requirements [Accessed 27 September 2017].

22 Kumar R, Gupta S, Kaur A, Gupta M. Individual donor-nucleic acid testing for human immunodeficiency virus-1, hepatitis $C$ virus and hepatitis B virus and its role in blood safety. Asian J Transfus Sci 2015;9:199-202.

23 Chigurupati P, Murthy KS. Automated nucleic acid amplification testing in blood banks: An additional layer of blood safety. Asian J Transfus Sci 2015;9:9-11.

24 Kleinman SH, Lelie N, Busch MP. Infectivity of human immunodeficiency virus-1, hepatitis $C$ virus, and hepatitis $B$ virus and risk of transmission by transfusion. Transfusion 2009;49:2454-89.

25 Laperche S. Blood safety and nucleic acid testing in Europe. Eurosurveillance 2005;10:pii=516.

26 The Penrose Inquiry. Final Report. Edinburgh: APS Group Scotland, 2015.

27 Anderson SA, Yang H, Gallagher LM et al. Quantitative estimate of the risks and benefits of possible alternative blood donor deferral strategies for men who have had sex with men. Transfusion 2009;49:1102-14.
28 Belanger GA, McFarland W, Raymond HF, Custer B. If the permanent deferral were lifted would men who have sex with men want to donate blood, and if so, who would be eligible? Transfusion 2013;53:2729-33.

29 Liszewski W, Terndrup C, Jackson NR, Helland S, Lavin BC. The beliefs and willingness of men who have sex with men to comply with a one-year blood donation deferral policy: a cross-sectional study. Transfusion 2017;57:2234-9.

30 Bednall TC, Bove LL, Cheetham A, Murray AL. A systematic review and meta-analysis of antecedents of blood donation behavior and intentions. Soc Sci Med 2013;96:86-94.

31 Grenfell P, Nutland W, McManus S et al. Views and experiences of men who have sex with men on the ban on blood donation: a cross sectional survey with qualitative interviews. BMJ 2011;343:d5604.

32 Seed CR, Lucky TT, Waller D et al. Compliance with the current 12-month deferral for male-to-male sex in Australia. Vox Sang 2013;106:14-22.

33 Offergeld R, Kamp C, Heiden M, Norda R, Behr-Gross M. Sexual risk behaviour and donor deferral in Europe. Vox Sang 2014;107:420-7.

34 Suligoi B, Pupella S, Regine V et al. Changing blood donor screening criteria from permanent deferral for men who have sex with men to individual sexual risk assessment: no evidence of a significant impact on the human immunodeficiency virus epidemic in Italy. Blood Transfus 2013;11:441-8.

35 Raimondo M, Facco G, Regine V et al. HIV-positive blood donors unaware of their sexual at-risk behaviours before donation in Italy. Vox Sang 2015;110:134-42.

36 Bes M, Piron M, Casamitjana N et al. Epidemiological trends of HIV-1 infection in blood donors from Catalonia, Spain (2005-2014). Transfusion 2017;57:2164-73.

37 Lieshout-Krikke RW, Zaaijer HL, van de Laar TJ. Predonation screening of candidate donors and prevention of window period donations. Transfusion 2014;55:373-8.

Address for correspondence: Ms Beattie RH Sturrock, under care of Dr Stuart Mucklow, Department of Clinical Haematology, Royal Berkshire NHS Foundation Trust, London Road, Reading RG1 5AN, UK. Email: beattie.sturrock@yahoo.co.uk 